\title{
Duality results for the joint spectral radius and transient behavior
}

\author{
Elmar Plischke* Fabian Wirth** Nikita Barabanov***
}

\begin{abstract}
For linear inclusions in discrete or continuous time several quantities characterizing the growth behavior of the corresponding semigroup are analyzed. These quantities are the joint spectral radius, the initial growth rate and (for bounded semigroups) the transient bound. It is recalled how these constants relate to one another and how they are characterized by various norms. A complete duality theory is developed in this framework, relating semigroups and dual semigroups and extremal or transient norms with their respective dual norms.
\end{abstract}

\section{INTRODUCTION}

In this paper we discuss duality relations between growth rates, i.e. joint spectral radii, initial growth rates and transient bounds of semigroups generated by linear inclusions. Our approach is based on the classical concept of a dual norm, see e.g. [17], as norms play a role in the description of all three quantities we are discussing

The joint spectral radius as introduced by Rota and Strang [1] characterizes the exponential growth rate of a linear semigroup generated by a compact set of matrices in discrete or continuous time, see also [2], [3]. One important tool in the study of the joint spectral radius consists in extremal norms, which characterize it. This study has begun with results by Barabanov [4], [2] and Kozyakin [5].

The initial growth rate is another quantity that characterizes an aspect of the exponential growth of a semigroup. It was introduced by Dahlquist [8] and Lozinskii [9] under the names "logarithmic norm" or "logarithmic derivative", Vidyasagar [16] uses the name "matrix measure". Its study has been motivated by problems in numerics, where the concept is used to obtain estimates for the accuracy of ODE solvers, or calculating the sensitivity of matrix exponentials.

Finally, the transient bound characterizes the overshoot of the semigroup. While the practical problem is classical, sharp bounds for the transient behavior are elusive and there is no complete theory on this issue. A number of results may be found in [10] and [11, Chapter 5]. It is known that the transient behavior may be characterized via appropriate norms. Also in this setting we develop a duality theory.

The main results of the paper concern duality notions between the different constants describing the growth of linear semigroups. To this end, we define for a given linear inclusion the corresponding dual inclusion. It is easy to see that joint spectral radius and transient bound for the dual semigroup are the same. Furthermore, two main results

\footnotetext{
* Zentrum für Technomathematik, Universität Bremen, Germany. elmar@math.uni-bremen.de

** Hamilton Institute, NUI Maynooth, Maynooth, Co. Kildare, Ireland Fabian. Wirthanuim. ie

*** North Dakota State University, Fargo, ND, USA nikita.barabanovandsu.edu
}

are obtained concerning dual norms: In the literature two specific constructions for extremal norms may be found, due to Barabanov [4], [2] and Protasov [12]. We show that these constructions are dual to one another in the sense, that if $v$ is a Barabanov norm for a semigroup, then the dual norm $v^{*}$ is a Protasov norm for the dual semigroup. A similar situation occurs for transient norms. In the literature there is a standard technique for constructing a norm that characterizes the transient bound, which to the best of our knowledge goes back to Feller, [13]. We show that there is a dual construction to Feller's, which appears to be new in this context. Again a norm is a Feller norm for a bounded semigroup if and only if its dual norm is of this dual type for the dual semigroup.

The paper is organized as follows. After introducing the basic concepts in Section II, in Section III we discuss initial growth rates for the continuous time case and give interpretation in terms of subgradients of norms. Section IV is devoted to some easy bounds for the transient behavior of linear semigroups. In Section V several important norms are introduced. Extremal norms are those that characterize the joint spectral radius, in that the initial growth rate is equal to the joint spectral radius with respect to these norms. Similarly, transient norms characterize the transient behavior in terms of their eccentricity, which we define below. Section VI is devoted to duality results concerning the joint spectral radius. Dual semigroups are introduced and it is shown that some construction procedures for extremal norms are dual. In Section VII similar results are obtained for transient norms.

For reasons of space some proofs had to be omitted. These will appear in a future journal publication.

\section{LINEAR INCLUSIONS}

In the following we study linear inclusions in continuous and discrete time. When necessary we specify the time set $\mathbb{T}$, which is thus either equal to $\mathbb{R}_{+}:=[0, \infty)$ or to $\mathbb{N}$.

Let $\mathbb{K}=\mathbb{R}, \mathbb{C}$. Given a compact set $\emptyset \neq \mathcal{M} \subset \mathbb{K}^{n \times n}$ and the time set $\mathbb{T}=\mathbb{N}$ we consider the discrete inclusion

$$
\begin{aligned}
x(t+1) & \in\{A x(t) \mid A \in \mathcal{M}\}, \quad t \in \mathbb{N} \\
x(0) & =x_{0} \in \mathbb{K}^{n} .
\end{aligned}
$$

A sequence $\{x(t)\}_{t \in \mathbb{N}}$ is called a solution of (1) with initial condition $x_{0}$ if $x(0)=x_{0}$ and if for all $t \in \mathbb{N}$ there exists an $A(t) \in \mathcal{M}$ such that $x(t+1)=A(t) x(t)$. Associated to (1) we consider the sets of products of length $t$ given by

$$
\mathcal{S}_{t}:=\{A(t-1) \ldots A(0) \mid A(s) \in \mathcal{M}, s=0, \ldots, t-1\},
$$

where we set $\mathcal{S}_{0}=\left\{I_{n}\right\}$ for $t=0$, and the semigroup given by $\mathcal{S}:=\bigcup_{t=0}^{\infty} \mathcal{S}_{t}$. 
In a similar manner we obtain a semigroup in the continuous time case. Given a compact set $\emptyset \neq \mathcal{M} \subset \mathbb{K}^{n \times n}$ and the time set $\mathbb{T}=\mathbb{R}_{+}$, we consider the semigroup generated by a differential inclusion

$$
\dot{x} \in\{A x(t) \mid A \in \mathcal{M}\} .
$$

A function $x: \mathbb{R}_{+} \rightarrow \mathbb{K}^{n}$ is called solution of (2) if it is absolutely continuous and satisfies $\dot{x}(t) \in\{A x(t) \mid A \in$ $\mathcal{M}\}$ almost everywhere. Equivalently, $x(\cdot)$ is the solution of a linear time-varying differential equation

$$
\dot{x}=A(t) x(t)
$$

for an appropriately chosen measurable map $A: \mathbb{R}_{+} \rightarrow \mathcal{M}$. We denote the evolution operators of (3) by $\Phi_{A}(t, s)$. The set of time $t$ transition operators is then given by

$$
\mathcal{S}_{t}:=\left\{\Phi_{A}(t, 0) \mid A: \mathbb{R}_{+} \rightarrow \mathcal{M} \text { measurable }\right\} .
$$

Again $\mathcal{S}=\bigcup_{t \in \mathbb{T}} \mathcal{S}_{t}$ defines a semigroup. In the sequel, we will tacitly assume that $\mathcal{S}$ is generated by an inclusion of the form (1) or of the form (2), if we speak of a semigroup $(\mathcal{S}, \mathbb{T})$. Moreover it is assumed, that $\mathcal{M}$ is convex if $\mathbb{T}=\mathbb{R}_{+}$. Together with our compactness assumption this ensures that the sets $\mathcal{S}_{t}, t \in \mathbb{T}$ are compact. Note that if $\mathbb{T}=\mathbb{R}_{+}$we have by classical relaxation results, that

$$
\operatorname{cl} \mathcal{S}_{t}(\mathcal{M})=\mathcal{S}_{t}(\operatorname{conv} \mathcal{M})
$$

so that by going over to the convex hull of $\mathcal{M}$ we do not alter the semigroup significantly.

In the following we wish to introduce several quantities that characterize the growth behavior of a semigroup $\mathcal{S}$. These are the joint spectral radius (or maximal Lyapunov exponent, or Lyapunov indicator), that characterizes the long term exponential growth behavior, the initial growth rate and the transient bound.

Remark 2.1: Whenever discrete time and continuous time systems are considered simultaneously, the dilemma appears that in discrete time it is natural to denote exponential growth in the form $r^{t}$, while in continuous time it of interest to consider $e^{\log r t}$. To keep notation short we have opted for a unified notation using the discrete time approach.

We begin our definitions with the joint spectral radius. Let $r(A)$ denote the spectral radius of $A$ and let $\|\cdot\|$ be some operator norm on $\mathbb{K}^{n \times n}$. Define for $t \in \mathbb{N}$

$$
\begin{gathered}
\bar{\rho}_{t}(\mathcal{M}):=\sup \left\{r(S)^{1 / t} \mid S \in \mathcal{S}_{t}\right\}, \\
\hat{\rho}_{t}(\mathcal{M}):=\sup \left\{\|S\|^{1 / t} \mid S \in \mathcal{S}_{t}\right\} .
\end{gathered}
$$

The joint spectral radius is defined by

$$
\rho(\mathcal{M}):=\limsup _{t \rightarrow \infty} \bar{\rho}_{t}(\mathcal{M})=\lim _{t \rightarrow \infty} \hat{\rho}_{t}(\mathcal{M}) .
$$

By the results in [14] the above quantity is well-defined. Note in particular that it does not depend on the choice of the norm $\|\cdot\|$. A further characterization of $\rho$ is given by

$\rho(\mathcal{M})=\inf \left\{\rho \in \mathbb{R} \mid \exists M_{\rho}: \forall t \in \mathbb{T}, S \in \mathcal{S}_{t}:\|S\| \leq M_{\rho} \rho^{t}\right\}$.
On the other hand, the initial growth rate of $\mathcal{M}$ depends on the norm under consideration. Given a norm $\|\cdot\|$ on $\mathbb{K}^{n}$ we define for the discrete time case $\mathbb{T}=\mathbb{N}$ the initial growth rate by

$$
\mu(\mathcal{M}):=\sup \{\|A\| \mid A \in \mathcal{M}\} .
$$

In the continuous time case $\mathbb{T}=\mathbb{R}_{+}$we set for an individual matrix $A \in \mathbb{K}^{n \times n}$

$$
\mu(A):=\exp \left(\lim _{t \rightarrow 0} \frac{1}{t} \log \left\|e^{A t}\right\|\right)
$$

and for $\mathcal{M} \subset \mathbb{K}^{n \times n}$ we let $\mu(\mathcal{M}):=\sup \{\mu(A) \mid A \in \mathcal{M}\}$.

If $\mu(A) \leq 1$ then $A$ is called dissipative. The following result extends statements in [16] and shows that $\mu$ is conceptually closely related to $\rho$, if we compare (5) with (8).

Proposition 2.1: Let $\mathbb{T}=\mathbb{N}, \mathbb{R}_{+}$. Suppose $\|\cdot\|$ is an operator norm on $\mathbb{K}^{n \times n}$. For $\mathcal{M} \subset \mathbb{K}^{n \times n}$ the initial growth rate $\mu(\mathcal{M})$ is the least upper exponential bound for $\|S\|$, i.e.,

$$
\mu(\mathcal{M})=\min \left\{\mu \in \mathbb{R} \mid \forall t \geq 0, S \in \mathcal{S}_{t}:\|S\| \leq \mu^{t}\right\} .
$$

Note, that from comparing (5) with (8) it is immediate, that for any norm $\|\cdot\|$ on $\mathbb{K}^{n}$ we have

$$
\rho(\mathcal{M}) \leq \mu_{\|\cdot\|}(\mathcal{M}) .
$$

For stable linear inclusions (those that generate a bounded semigroup) we define the transient bound by

$$
M_{0}(\mathcal{M}):=\sup \{\|S\| \mid S \in \mathcal{S}(\mathcal{M})\} .
$$

In particular, if $\mathcal{M}$ is a set of dissipative matrices then the semigroup $\mathcal{S}(\mathcal{M})$ generated by $\mathcal{M}$ is a contraction semigroup with $M_{0}(\mathcal{M})=1$.

In Section VI we will discuss the interplay of $\rho, \mu$ and $M_{0}$ with dual semigroups. Before doing so, we discuss initial growth rates in more detail.

\section{INITIAL GROWTH RATES}

In this section we review some known results on initial growth rates. We also present a characterization of initial growth rates in terms of subgradients of the norm. While this characterization is not difficult or surprising from the point of view of nonsmooth analysis, the remark does not appear to have been made in this context.

We now discuss the characterization of initial growth rates using subgradients. To this end we need subgradients of norms, which are given through the dual norm. Recall that for a fixed norm $v$ on $\mathbb{K}^{n}$ the dual norm is defined by

$$
v^{*}(x):=\max \{|\langle l, x\rangle| \mid v(l) \leq 1\} .
$$

Note that $v=\left(v^{*}\right)^{*}$. A pair of vectors $l, x \in \mathbb{K}^{n}$ is called dual pair, if $\langle l, x\rangle=v(x) v^{*}(l)$. See [17] for further details.

Let us denote the unit sphere of the vector norm $v$ by $\mathcal{T}_{v}=\left\{x \in \mathbb{K}^{n} \mid v(x)=1\right\}$ and the unit sphere of its dual norm by $\mathcal{T}_{v}^{*}$. We want to relate the initial growth rate to pairs of dual vectors. To this end we need to recall the concept of a subdifferential of a convex function. Given a convex function $f: \mathbb{K}^{n} \rightarrow \mathbb{R}$ the subdifferential $\partial_{P} f(x)$ is defined by

$\partial_{P} f(x):=\left\{l \in \mathbb{K}^{n} \mid f(y) \geq f(x)+\operatorname{Re}\langle l, y-x\rangle, \forall y \in \mathbb{K}^{n}\right\}$. 
We see that for a norm $v$ on $\mathbb{K}^{n}$ that the elements of $\partial_{P} v(x)$ are normals for supporting hyperplanes in $x$ of the convex set $v(x) \mathcal{B}_{v}:=\left\{y \in \mathbb{K}^{n} \mid v(y) \leq v(x)\right\}$. It follows from [18, Corollary 23.5.3] that

$$
\partial_{P} v(x)=\left\{l \in \mathbb{K}^{n} \mid v^{*}(l)=1,\langle l, x\rangle=v(x)\right\} .
$$

Proposition 3.1: Let $\mathbb{T}=\mathbb{R}_{+}$. Given a norm $v$ on $\mathbb{K}^{n}$, the associated initial growth rate $\mu_{v}$ of a matrix $A$ is given by

$$
\begin{aligned}
\log \mu_{v}(A)= & \max \{\operatorname{Re}\langle l, A x\rangle \\
& \left.\mid(l, x) \in \mathcal{T}_{v}^{*} \times \mathcal{T}_{v} \text { is a dual pair of } v\right\} .
\end{aligned}
$$

For $A \in \mathbb{K}^{n \times n}$ we denote by $A^{*}$ the dual matrix with respect to the standard scalar product, that is, $A^{*}=\bar{A}^{T}$. Proposition 3.1 can be used to derive, in a simple manner, a (well-known) formula for the initial growth rate $\mu_{2}$ with respect to the Euclidean norm.

Lemma 3.1: The Euclidean initial growth rate of $A \in$ $\mathbb{K}^{n \times n}$ is given by $\log \mu_{2}(A)=\frac{1}{2} \lambda_{\max }\left(A+A^{*}\right)$.

Proof: For the Euclidean norm all vectors are self-dual, as $\langle x, x\rangle=\|x\|_{2}^{2}$. Hence

$$
\begin{aligned}
\log \mu_{2}(A) & =\max \left\{\operatorname{Re}\langle x, A x\rangle \mid\|x\|_{2}=1\right\} \\
& =\frac{1}{2} \max _{x \neq 0} \frac{\left\langle x,\left(A+A^{*}\right) x\right\rangle}{\langle x, x\rangle}=\frac{1}{2} \lambda_{\max }\left(A+A^{*}\right)
\end{aligned}
$$

which follows from the Rayleigh-Ritz theorem, see [17].

\section{BOUNDS FOR THE TRANSIENT BEHAVIOR}

In this section we discuss how to obtain transient bounds via the initial growth rate. To this end we introduce the eccentricity to be able to compare two norms.

Definition 4.1: Suppose $v$ and $\|\cdot\|$ are norms on $\mathbb{K}^{n}$. The eccentricity of $v(\cdot)$ with respect to $\|\cdot\|$ is given by

$$
\operatorname{ecc}(v)=\operatorname{ecc}(v,\|\cdot\|):=\frac{\max _{\|x\|=1} v(x)}{\min _{\|x\|=1} v(x)} .
$$

The eccentricity measures the deformation of the unit balls of two norms w.r.t. each other. It is easy to see that

$$
\operatorname{ecc}(v,\|\cdot\|)=\operatorname{ecc}(\|\cdot\|, v) \text {. }
$$

This notion can be used to compare the transient behavior under different norms.

Corollary 4.1: Given $\mathcal{M} \subset \mathbb{K}^{n \times n}$ and two norms $\|\cdot\|, v(\cdot)$ on $\mathbb{K}^{n}$. Then for all $S \in \mathcal{S}_{t}(\mathcal{M}, \mathbb{T})$ we have

$$
\|S\| \leq \operatorname{ecc}(v,\|\cdot\|) \mu_{v}(\mathcal{M})^{t}, \quad t \in \mathbb{T} .
$$

\section{EXTREMAL AND TRANSIENT NORMS}

Both joint spectral radius and transient bound are closely related to specific norms.

Definition 5.1: Let $\mathbb{K}=\mathbb{R}, \mathbb{C}, \mathbb{T}=\mathbb{N}, \mathbb{R}_{+}$and let $(\mathcal{S}, \mathbb{T})$ be a semigroup in $\mathbb{K}^{n \times n}$.

(i) A norm $v$ on $\mathbb{K}^{n}$ is called extremal for $\mathcal{S}$ if

$$
\mu_{v}(\mathcal{S})=\rho(\mathcal{S}) .
$$

(ii) an extremal norm $v$ on $\mathbb{K}^{n}$ is called Barabanov norm corresponding to $\mathcal{S}$ if for all $x \in \mathbb{K}^{n}, t \in \mathbb{T}$ there is an $S \in \operatorname{cl} \mathcal{S}_{t}$ such that

$$
v(S x)=\rho(\mathcal{S})^{t} v(x) .
$$

(iii) a norm $v$ on $\mathbb{K}^{n}$ is called Protasov norm corresponding to $\mathcal{S}$ if the unit ball $\mathcal{B}_{v}$ of $v$ satisfies

$$
\rho(\mathcal{S})^{t} \mathcal{B}_{v}=\operatorname{conv} \operatorname{cl} \mathcal{S}_{t} \mathcal{B}_{v}, \quad \forall t \in \mathbb{T} .
$$

Remark 5.1: It has become common to use the name Barabanov norms because they have been introduced in [4], [2]. A sufficient criterion for their existence is that $\mathcal{M}$ is irreducible, i.e., only the trivial subspaces $\{0\}$ and $\mathbb{K}^{n}$ are invariant under all matrices $A \in \mathcal{M}$. It is clear that Barabanov norms are extremal. The converse is false.

In a similar vein, we use the name Protasov norm, because these norms have been introduced in [12]. It is easy to see directly that they are extremal. This also follows from the duality result Theorem 6.1 .

The next lemma provides a construction for Protasov norms.

Lemma 5.1: Let $\mathbb{K}=\mathbb{R}, \mathbb{C}, \mathbb{T}=\mathbb{N}, \mathbb{R}_{+}$and let $(\mathcal{S}, \mathbb{T})$ be an irreducible semigroup in $\mathbb{K}^{n \times n}$. If $v$ is an extremal norm on $\mathbb{K}^{n}$ with unit ball $\mathcal{B}$ then

$$
\tilde{\mathcal{B}}=\bigcap_{t \in \mathbb{T}} \operatorname{conv} \operatorname{cl}\left\{\rho(\mathcal{S})^{-t} S \mathcal{B} \mid S \in \operatorname{cl} \mathcal{S}_{t}\right\}
$$

is the unit ball of a Protasov norm for $\mathcal{S}$.

Proof: We may assume $\rho(\mathcal{S})=1$. Obviously, $\tilde{\mathcal{B}}$ is convex, balanced and closed. It is not contained in any hyperplane, because the semigroup is irreducible. From the definition it follows that $\tilde{\mathcal{B}}=\operatorname{conv} \operatorname{cl} \mathcal{S}_{t} \tilde{\mathcal{B}}, \forall t \in \mathbb{T}$. Hence, $\tilde{\mathcal{B}}$ is a level set of a Protasov norm.

Definition 5.2: Let $\mathbb{K}=\mathbb{R}, \mathbb{C}, \mathbb{T}=\mathbb{N}, \mathbb{R}_{+}$and let $(\mathcal{S}, \mathbb{T})$ be a bounded semigroup in $\mathbb{K}^{n \times n}$ generated by $\mathcal{M} \subset \mathbb{K}^{n \times n}$. Consider a fixed vector norm $\|\cdot\|$.

(i) A norm $v$ on $\mathbb{K}^{n}$ is called transient for $\mathcal{S}$ if

$$
\mu_{v}(\mathcal{M}) \leq 1, \quad \text { and } \operatorname{ecc} v,\|\cdot\|=M_{0}(\mathcal{M}) .
$$

(ii) The Feller norm corresponding to $\mathcal{M}$ and $\|\cdot\|$ is defined by

$$
\|x\|_{\mathcal{M}}=\sup _{S \in \mathcal{S}}\|S x\|
$$

(iii) The convex-transient norm corresponding to $\mathcal{M}$ and $\|\cdot\|$ is defined by its unit ball given by

$$
\tilde{\mathcal{B}}_{\mathcal{M}}=\operatorname{conv} \operatorname{cl}\{S x \mid S \in \mathcal{S}, x \in \mathcal{B}\}=\operatorname{conv} \operatorname{cl} \bigcup_{S \in \mathcal{S}} S \mathcal{B}
$$

where $\mathcal{B}$ is the unit ball of $\|\cdot\|$.

Remark 5.2: The name transient norm comes from the fact that the eccentricity of the norm characterizes the transient bound. We opted for the name Feller norm as the norm $x \mapsto \sup _{t>0}\left\|T_{t} x\right\|$ for a semigroup $\left(T, \mathbb{R}_{+}\right)$has been introduced in [13]. Note that $\mu_{v}(\mathcal{M}) \leq 1$ implies for any norm $v$ that $\operatorname{ecc}(v,\|\cdot\|) \geq M_{0}(\mathcal{M})$. We will now show that the Feller norm $\|\cdot\|_{\mathcal{M}}$ is a transient norm. The same statement for the convex-transient norm follows from our duality results.

It is easily verified that $\|\cdot\|_{\mathcal{M}}$ is indeed a norm. We claim that ecc $\|\cdot\|_{\mathcal{M}}=M_{0}(\mathcal{M})$. This may be seen as follows:

$$
\operatorname{ecc}\left(\|\cdot\|_{\mathcal{M}},\|\cdot\|\right)=\frac{\sup _{\|x\|=1} \sup _{S \in \mathcal{S}}\|S x\|}{\inf _{\|x\|=1} \sup _{S \in \mathcal{S}}\|S x\|},
$$


but $\inf _{\|x\|=1} \sup _{S \in \mathcal{S}}\|S x\|=1$ because otherwise $\mathcal{S}$ is not bounded. Furthermore, $\mu_{\mathcal{M}}(\mathcal{M}) \leq 1$ because

$$
\|S x\|_{\mathcal{M}}=\sup _{T \in \mathcal{S}}\|T S x\| \leq \sup _{T \in \mathcal{S}}\|T x\|=\|x\|_{\mathcal{M}} .
$$

More precisely, we have the following result for the initial growth rate with respect to the transient norm.

Lemma 5.2: Let $\mathcal{M} \subset \mathbb{K}^{n \times n}$ generate a bounded semigroup $\mathcal{S}$. Then the initial growth rate associated with the Feller norm satisfies $\mu_{\mathcal{M}}(\mathcal{M})=\min \{\mu(\mathcal{M}), 1\}$.

Proof: If for the original norm $\mu(\mathcal{M}) \leq 1$, then, by Proposition 2.1, $\|S x\| \leq \mu(\mathcal{M})^{t}\|x\| \leq\|x\|$ for all $x \in \mathbb{K}^{n}$ and all $S \in \mathcal{S}_{t}$. Hence $\|\cdot\|_{\mathcal{M}}=\sup _{S \in \mathcal{S}}\|S \cdot\|=\|\cdot\|$ and so $\mu_{\mathcal{M}}(\mathcal{M})=\mu(\mathcal{M})$.

Now, if $\mu(\mathcal{M})>1$ there exist $x_{0} \in \mathbb{K}^{n}$ and $S \in \mathcal{S}_{t_{0}}, t_{0}>$ 0 such that $\left\|S x_{0}\right\|=\left\|x_{0}\right\|_{\mathcal{M}}>\left\|x_{0}\right\|$. By Proposition 2.1 this shows $\mu_{\mathcal{M}}(\mathcal{M}) \geq 1$ and so $\mu_{\mathcal{M}}(\mathcal{M})=1$ by (21).

We note the following property of the unit ball of the Feller norm for further reference.

Lemma 5.3: Suppose that $\mathcal{M} \subset \mathbb{K}^{n \times n}$ generates a bounded semigroup. Then the unit ball $\mathcal{B}_{\mathcal{M}}$ of the associated Feller norm $\|\cdot\|_{\mathcal{M}}$ is given by

$$
\mathcal{B}_{\mathcal{M}}=\bigcap_{S \in \mathcal{S}} S^{-1} \mathcal{B}
$$

where $\mathcal{B}$ is the unit ball of $\|\cdot\|$.

Proof: The set $\bigcap_{S \in \mathcal{S}} S^{-1} \mathcal{B}$ is a closed convex balanced set. Also, 0 is contained in the interior of this set by the boundedness of $\mathcal{S}$. Therefore $\bigcap_{S \in \mathcal{S}} S^{-1} \mathcal{B}$ is the unit ball of a norm. By definition, $x \in \mathcal{B}_{\mathcal{M}}$ holds if and only if for all $S \in$ $\mathcal{S}, S x \in \mathcal{B}$, or equivalently, $x \in S^{-1} \mathcal{B}$ which yields (22). Finally, we note that if $\rho(\mathcal{M})=1$ and $\mathcal{M}$ is bounded, then the Feller norm and the convex-transient norm are also extremal for $\mathcal{M}$, but in general different from a Barabanov or Protasov norm. Thus another way to construct extremal norms for irreducible inclusions, is to define the Feller or convex-transient norm for the normalized semigroup given by the finite time sets $\rho(\mathcal{M})^{-t} \mathcal{S}_{t}(\mathcal{M})$.

\section{DuAlity}

In this section we investigate the dual of semigroups. Also duality properties of Barabanov and Protasov norms as well as Feller and convex-transient norms are shown.

Let $\mathbb{K}=\mathbb{R}, \mathbb{C}$ and $\mathbb{T}=\mathbb{N}, \mathbb{R}_{+}$. Given a semigroup $(\mathcal{S}, \mathbb{T}) \subset \mathbb{K}^{n \times n}$, we define the dual semigroup to be

$$
\mathcal{S}^{*}:=\left\{S^{*} \mid S \in \mathcal{S}\right\},
$$

where we assume in particular that we have

$$
\left(\mathcal{S}^{*}\right)_{t}=\left\{S^{*} \mid S \in \mathcal{S}_{t}\right\}, \quad t \in \mathbb{T} .
$$

It is then immediate that $\rho(\mathcal{S})=\rho\left(\mathcal{S}^{*}\right)$. Let us briefly discuss how the generating sets can be constructed.

In the case $\mathbb{T}=\mathbb{N}$ let $\mathcal{S}$ be the semigroup generated by $(\mathcal{M}, \mathbb{N})$. Then $\mathcal{S}^{*}$ is generated by $\left(\mathcal{M}^{*}, \mathbb{N}\right)$, where we define

$$
\mathcal{M}^{*}:=\left\{A^{*} \mid A \in \mathcal{M}\right\} \text {. }
$$

In the continuous time case $\mathbb{T}=\mathbb{R}_{+}$, on the other hand, we consider the differential inclusion

$$
\dot{x}(t) \in\left\{A^{*}(t) \mid A \in \mathcal{M}\right\} .
$$

It is well known that for every $t \geq 0$ the evolution operators $\Phi(t, 0)$ of (3) of the form

$$
\Phi(t, 0)=e^{A_{k} t_{k}} e^{A_{k-1} t_{k-1}} \cdots e^{A_{1} t_{1}},
$$

where $A_{j} \in \mathcal{M}, j=1, \ldots, k, \sum_{j=1}^{k} t_{j}=t$, lie dense in $\mathcal{S}_{t}\left(\mathcal{M}, \mathbb{R}_{+}\right)$. It is obvious, that the dual of these operators lies dense in $\mathcal{S}_{t}\left(\mathcal{M}^{*}, \mathbb{R}_{+}\right)$, so $\operatorname{cl} \mathcal{S}_{t}\left(\mathcal{M}, \mathbb{R}_{+}\right)^{*}=\operatorname{cl} \mathcal{S}_{t}\left(\mathcal{M}^{*}, \mathbb{R}_{+}\right)$.

Thus we see that in both discrete and continuous time we may consider $\mathcal{M}^{*}$ to be the generator of $\mathcal{S}^{*}$.

Our first duality result is the following, see also [2].

Lemma 6.1: Let $\mathbb{K}=\mathbb{R}, \mathbb{C}, \mathbb{T}=\mathbb{N}, \mathbb{R}_{+}$. Let $w$ be an extremal norm for $(\mathcal{S}, \mathbb{T})$. Then $w^{*}$ is extremal for $\left(\mathcal{S}^{*}, \mathbb{T}\right)$.

We now show that Barabanov norms and Protasov norms are dual concepts.

Theorem 6.1: Let $\mathbb{K}=\mathbb{R}, \mathbb{C}, \mathbb{T}=\mathbb{N}, \mathbb{R}_{+}$and let $(\mathcal{S}, \mathbb{T})$ be a semigroup. The norm $v$ is a Barabanov norm for $(\mathcal{S}, \mathbb{T})$ if and only if the dual norm $v^{*}$ is a Protasov norm for $\left(\mathcal{S}^{*}, \mathbb{T}\right)$.

Proof: We may assume that $\rho(\mathcal{S})=1$. Assume furthermore that $v$ is a Barabanov norm and let $t \in \mathbb{T}$ and $x \in \mathbb{K}^{n}, v(x)=1$ be arbitrary. By assumption there exists an $S \in \mathcal{S}_{t}$ such that $v(S x)=v(x)=1$. As $v=v^{*^{*}}$ it follows that

$$
\begin{aligned}
v(S x) & =v(x)=\max \left\{\operatorname{Re}\langle l, x\rangle \mid v^{*}(l) \leq 1\right\} \\
& =\max \left\{\operatorname{Re}\left\langle S^{*} l, x\right\rangle \mid v^{*}(l) \leq 1\right\} \\
& \leq \max \left\{\operatorname{Re}\langle l, x\rangle \mid l \in \operatorname{conv} \mathcal{S}_{t}^{*} \mathcal{B}_{v^{*}}\right\} .
\end{aligned}
$$

On the other hand by the extremality of the norm $v^{*}$ we have $v(x) \geq \max \left\{\operatorname{Re}\langle l, x\rangle \mid l \in \operatorname{conv} \mathcal{S}_{t}^{*} \mathcal{B}_{v^{*}}\right\}$. This implies equality throughout the calculation. As $x$ was arbitrary this means that $v$ is the dual norm of the norm with the unit ball conv $\mathcal{S}_{t}^{*} \mathcal{B}_{v^{*}}$. As the dual norm is uniquely defined this implies that conv $\mathcal{S}_{t}^{*} \mathcal{B}_{v^{*}}$ is the unit ball of $v^{*}$ and as $t \in \mathbb{T}$ was arbitrary, condition (17) is satisfied. Hence, $v^{*}$ is a Protasov norm.

Conversely, assume that (17) holds for $v^{*}$ and let $t \in \mathbb{T}$ and $x \in \mathbb{K}^{n}, v(x)=1$ be arbitrary. Then

$$
\begin{aligned}
v(x) & =\max \left\{\operatorname{Re}\langle l, x\rangle \mid v^{*}(l) \leq 1\right\} \\
& =\max \left\{\operatorname{Re}\langle l, x\rangle \mid l \in \operatorname{conv} \mathcal{S}_{t}^{*} \mathcal{B}_{v^{*}}\right\} \\
& =\max \left\{\operatorname{Re}\left\langle S^{*} l, x\right\rangle \mid v^{*}(l) \leq 1, S^{*} \in \mathcal{S}_{t}^{*}\right\} \\
& =\max \left\{\operatorname{Re}\langle l, S x\rangle \mid v^{*}(l) \leq 1, S \in \mathcal{S}_{t}\right\} \\
& =\max \left\{v(S x) \mid S \in \mathcal{S}_{t}\right\},
\end{aligned}
$$

which shows that $v$ is a Barabanov norm.

A particularly satisfying situation occurs in the case that $\mathcal{S}=\mathcal{S}^{*}$ as in this case there is a natural candidate for an extremal norm.

Proposition 6.1: Let $\mathbb{K}=\mathbb{R}, \mathbb{C}, \mathbb{T}=\mathbb{N}, \mathbb{R}_{+}$and let $(\mathcal{S}, \mathbb{T})$ be an irreducible semigroup with $\mathcal{S}=\mathcal{S}^{*}$ then the Euclidean norm $\|\cdot\|_{2}$ is an extremal norm for $\mathcal{S}$.

Proof: Without loss of generality we may assume $\rho(\mathcal{S})=1$. Assume the assertion is false, then for some 
$x \in \mathbb{K}^{n},\|x\|_{2}=1$ and some $t \in \mathbb{T}$ there is an $S \in \mathcal{S}_{t}$ such that $\|S x\|_{2}>1$. As $S^{*} \in \mathcal{S}$ we have $S^{*} S \in \mathcal{S}$ and $r\left(S^{*} S\right) \geq\left\langle S^{*} S x, x\right\rangle=\|S x\|_{2}^{2}>1$. This contradicts $\rho(\mathcal{S})=1$ and the contradiction proves the assertion.

In the continuous time case $\mathbb{T}=\mathbb{R}_{+}$we also point out the following reformulation of a result by Barabanov that will turn out to be useful later. Here we relate for the continuous time case extremality properties of a norm with the infinitesimal growth of the trajectories of the system.

Proposition 6.2: Let $\mathbb{K}=\mathbb{R}, \mathbb{C}, \mathbb{T}=\mathbb{R}_{+}$and let $\left(\mathcal{S}, \mathbb{R}_{+}\right)$ be an irreducible semigroup generated by $\mathcal{M} \in I\left(\mathbb{K}^{n \times n}\right)$.

(i) A norm $v$ is an extremal norm for $\mathcal{S}$ if and only if for all dual pairs $l, x \in \mathbb{K}^{n}$ and all $A \in \mathcal{M}$ it holds that

$$
\operatorname{Re}\langle l, A x\rangle \leq \log \rho(\mathcal{M}) v(x) v^{*}(l) .
$$

In this case, there exist dual pairs $l, x \in \mathbb{K}^{n}$ and $A \in \mathcal{M}$ where equality in (24) is attained.

(ii) A norm $v$ is a Barabanov norm for $\mathcal{S}$ if and only if for all $x \in \mathbb{K}^{n}$ there exists an $l \in \mathbb{K}^{n}$ such that $l, x$ is a dual pair and an $A \in \mathcal{M}$ such that

$$
\operatorname{Re}\langle l, A x\rangle=\log \rho(\mathcal{M}) v(x) v^{*}(l) .
$$

The following result is a counterpart of Proposition 6.2 for the discrete-time case [4]. We assume that all matrices in the set $\mathcal{M}$ are nonsingular.

Proposition 6.3: Let $\mathbb{K}=\mathbb{R}, \mathbb{C}, \mathbb{T}=\mathbb{N}$ and let $(\mathcal{S}, \mathbb{N})$ be an irreducible semigroup generated by $\mathcal{M} \in I\left(\mathbb{K}^{n \times n}\right)$.

(i) A norm $v$ is an extremal norm for $\mathcal{S}$ if and only if for all pairs $l, x \in \mathbb{K}^{n}$ and all $A \in \mathcal{M}$ it holds that

$$
\operatorname{Re}\langle l, A x\rangle \leq \rho(\mathcal{M}) v(x) v^{*}(l) .
$$

In this case, there exist pairs $l, x \in \mathbb{K}^{n}$ and $A \in \mathcal{M}$ where equality in (26) is attained.

(ii) A norm $v$ is a Barabanov norm for $\mathcal{S}$ if and only if for all $x \in \mathbb{K}^{n}$ there exists an $l \in \mathbb{K}^{n}$ and an $A \in \mathcal{M}$ such that

$$
\operatorname{Re}\langle l, A x\rangle=\rho(\mathcal{M}) v(x) v^{*}(l) .
$$

Example 6.1: Consider the case $\mathbb{T}=\mathbb{N}$. Let $A_{1}=$ $\operatorname{diag}(0.5,1), A_{2}=S A_{1} S^{-1}$, where $S=\left(\begin{array}{cc}\cos \varphi & -\sin \varphi \\ \sin \varphi & \cos \varphi\end{array}\right)$ is a rotation by angle $\varphi$. Let us take $\varphi=\frac{\pi}{4}$ for convenience. $A_{1}$ is an orthogonal contraction with respect to the $y$-axis, while $A_{2}=\frac{1}{4}\left(\begin{array}{cc}3 & -1 \\ -1 & 3\end{array}\right)$ is an orthogonal contraction with respect to the axis $y=-x$.

The set $\mathcal{M}=\left\{A_{1}, A_{2}\right\}$ is irreducible (i.e. it has no common nontrivial subspace). Note also that $\mathcal{M}=\mathcal{M}^{*}$, so that by Theorem 6.1 the dual of a Barabanov norm for $\mathcal{M}$ is a Protasov norm for $\mathcal{M}^{*}=\mathcal{M}$. It is easy to see that the joint spectral radius satisfies $\rho(\mathcal{M})=1$. The unit ball $\mathcal{B}$ of a Barabanov norm is given by a parallelogram spanned by the vertices $\left\{\left(\begin{array}{l}0 \\ 1\end{array}\right),\left(\begin{array}{c}2 \\ 1\end{array}\right),\left(\begin{array}{c}0 \\ -1\end{array}\right),\left(\begin{array}{c}-2 \\ -1\end{array}\right)\right\}$. Its dual ball $\tilde{\mathcal{B}}$ is a parallelogram spanned by the vertices $\left\{\left(\begin{array}{c}1 \\ -1\end{array}\right),\left(\begin{array}{l}0 \\ 1\end{array}\right),\left(\begin{array}{c}-1 \\ 1\end{array}\right),\left(\begin{array}{c}0 \\ -1\end{array}\right)\right\}$. As $A_{1}\left(\begin{array}{l}0 \\ 1\end{array}\right)=\left(\begin{array}{l}0 \\ 1\end{array}\right)$ and $A_{2}\left(\begin{array}{c}1 \\ -1\end{array}\right)=$ $\left(\begin{array}{c}1 \\ -1\end{array}\right)$ we have conv $\left\{A_{1} \tilde{\mathcal{B}}, A_{2} \tilde{\mathcal{B}}\right\}=\tilde{\mathcal{B}}$, hence it is the norm ball of a Protasov norm for $\mathcal{M}$, see Fig. 6.1 for an illustration.

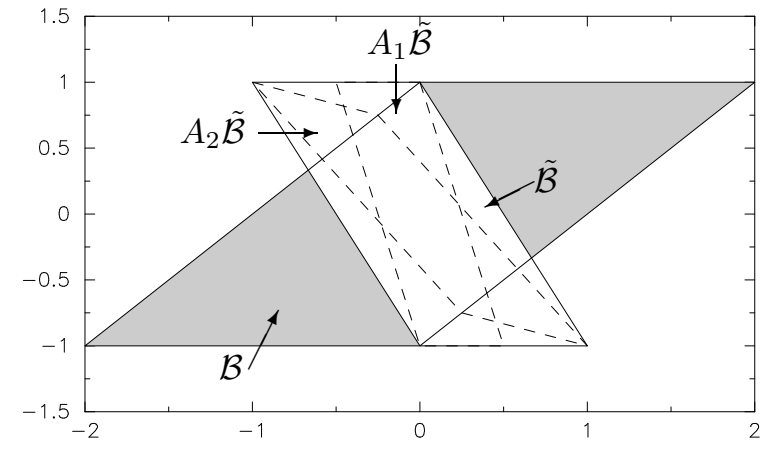

Fig. 1. Extremal norms of Example 6.1

\section{TRANSIENT NORMS AND DUALITY}

Let us now investigate duality issues for transient norms and initial growth rates. We introduce one further notation. If $M_{0}(\mathcal{M})$ is the transient bound of $\mathcal{S}(\mathcal{M})$ with respect to the norm $\|\cdot\|$, then we denote by $M_{0}^{*}(\mathcal{M})$ the transient bound with respect to the dual norm, i.e.

$$
M_{0}^{*}(\mathcal{M})=\sup \left\{\|S\|^{*} \mid S \in \mathcal{S}\right\} .
$$

For dual norms we obtain the following result.

Theorem 7.1: Suppose that $\|\cdot\|$ is a vector norm on $\mathbb{K}^{n}$ with associated initial growth rate $\mu(\cdot)$ and let $\mu^{*}(\cdot)$ denote the initial growth rate with respect to the dual norm $\|\cdot\|^{*}$ on $\mathbb{K}^{n}$. Then for a set of matrices $\mathcal{M} \subset \mathbb{K}^{n \times n}$ the following statements hold

1) $M_{0}(\mathcal{M})=M_{0}^{*}\left(\mathcal{M}^{*}\right)$,

2) $\mu(\mathcal{M})=\mu^{*}\left(\mathcal{M}^{*}\right)$.

3) $\log \mu_{2}(\mathcal{M}) \leq \frac{1}{2}\left(\log \mu(\mathcal{M})+\log \mu^{*}(\mathcal{M})\right)$.

Proof: The first statement is obvious. We prove the remaining statements for the case $\mathbb{T}=\mathbb{R}_{+}$, the discrete time case follows in a similar manner. It follows from Proposition 3.1 that for all $A \in \mathcal{M}$

$$
\begin{aligned}
\log \mu(A) & =\max _{\|x\|=1} \max _{\|l\|^{*}=1,\langle l, x\rangle=1} \operatorname{Re}\langle l, A x\rangle, \\
\log \mu^{*}\left(A^{*}\right) & =\max _{\|l\|^{*}=1} \max _{\|x\|=1,\langle l, x\rangle=1} \operatorname{Re}\left\langle x, A^{*} l\right\rangle .
\end{aligned}
$$

Now as $\operatorname{Re}\langle l, A x\rangle=\operatorname{Re}\left\langle x, A^{*} l\right\rangle$ the equality $\mu^{*}(A)=$ $\mu\left(A^{*}\right)$ is proved. The final statement follows from the second, because

$$
\begin{aligned}
\log \mu(A)+\log \mu^{*}(A) & =\log \mu(A)+\log \mu\left(A^{*}\right) \\
& \geq \log \mu\left(A+A^{*}\right) \geq \alpha\left(A+A^{*}\right) \\
& =\lambda_{\max }\left(A+A^{*}\right)=2 \log \mu_{2}(A)
\end{aligned}
$$

where we used that $\log \mu(B)$ is a convex function, which is bounded from below by the spectral abscissa $\alpha(B)=$ $\max \operatorname{Re} \sigma(B)$, see [16]. In case of a Hermitian matrix $B=$ $A+A^{*}$ this abscissa is an eigenvalue. By Lemma 3.1 this eigenvalue equals $\log \mu_{2}(A)$. Clearly, $\log \mu(A)+$ $\log \mu\left(A^{*}\right) \leq \log \mu(\mathcal{M})+\log \mu\left(\mathcal{M}^{*}\right)$ for all $A \in \mathcal{M}$.

This theorem shows that the initial growth rate for the spectral norm is the best lower bound for all mean values of dual initial growth rates. Especially, for the the dual 1- and $\infty$-norms we immediately obtain $\mu_{1}^{*}(\mathcal{M})=\mu_{\infty}(\mathcal{M})$ for all 
$\mathcal{M} \subset \mathbb{K}^{n \times n}$. Formulas for these initial growth rates are well known, see [16]. Using these formulas part 2 of Theorem 7.1 implies

Corollary 7.1: Suppose that $\mathcal{M} \subset \mathbb{K}^{n \times n}$ is a set of column or row diagonally dominant matrices with $\log \mu_{1}(A)+$ $\log \mu_{\infty}(A)<0$ for all $A \in \mathcal{M}$. Then $\mathcal{S}(\mathcal{M})$ satisifies $\log \mu_{2}(\mathcal{M}) \leq 0$.

Now that we have treated the initial growth of dual norms we proceed to the second main result of this paper. Feller norms and convex-transient norms are dual concepts. To this end we first need the following property of the eccentricity.

Proposition 7.1: For norms $v,\|\cdot\|$ on $\mathbb{K}^{n}$ it holds that

$$
\operatorname{ecc}(v,\|\cdot\|)=\operatorname{ecc}\left(v^{*},\|\cdot\|^{*}\right) \text {. }
$$

Proof: By symmetry and using (13) it is sufficient to show that $\max _{\|y\|^{*}=1} v^{*}(y)=\max _{v(x)=1}\|x\|$ and $\min _{\|y\|^{*}=1} v^{*}(y) \geq \min _{v(x)=1}\|x\|$. To show the first of these claims note that by definition,

$$
\max _{\|y\|^{*}=1} v^{*}(y)=\max _{v(x)=1} \max _{\|y\|^{*}=1}\left|y^{*} x\right|=\max _{v(x)=1}\|x\| .
$$

To show the second claim, assume that $\alpha \in \mathbb{R}_{+}$is maximal with the property $v(\alpha z) \leq 1$ for all $\|z\|=1$. Setting $u=\alpha z$ we have $\alpha=\min _{v(u)=1}\|u\|$. Then

$$
\begin{array}{r}
\min _{\|y\|^{*}=1} v^{*}(y)=\min _{\|y\|^{*}=1} \max _{v(x) \leq 1}\left|y^{*} x\right| \\
\geq \min _{\|y\|^{*}=1} \alpha\left|y^{*} z\right| \\
=\alpha=\min _{v(u)=1}\|u\|
\end{array}
$$

where we replaced $x$ by $\alpha z$ such that $z$ satisfies $\nu(\alpha z) \leq 1$, $\|z\|=1$ and $y^{*} z=\|y\|^{*}$. Combining (28), (29) and (13), we obtain

$\operatorname{ecc}\left(v^{*},\|\cdot\|^{*}\right) \leq \frac{\max _{v(y)=1}\|y\|}{\min _{v(y)=1}\|y\|}=\operatorname{ecc}(\|\cdot\|, v)=\operatorname{ecc}(v,\|\cdot\|)$.

By symmetry we obtain equality throughout.

By Proposition 7.1 the dual norm of a transient norm satisfies $\operatorname{ecc}\left(v^{*}\right)=\operatorname{ecc}(v)$ when $\|\cdot\|$ is the Euclidean norm. Let us therefore consider the norm $w(\cdot):=\left(v(\cdot)_{\mathcal{M}^{*}}^{*}\right)^{*}$ which has the same eccentricity as the Feller norm $v(\cdot)_{\mathcal{M}^{*}}^{*}$ Then $w$ coincides with the convex-transient norm.

Theorem 7.2: Let $\mathbb{T}=\mathbb{R}_{+}, \mathbb{N}, \mathbb{K}=\mathbb{R}, \mathbb{C}$. Assume that $\mathcal{M} \subset \mathbb{K}^{n \times n}$ generates a bounded semigroup $(\mathcal{S}, \mathbb{T})$. Then the unit ball $\tilde{\mathcal{B}}_{\mathcal{M}}$ of the convex-transient norm satisfies

$$
\left(\tilde{\mathcal{B}}_{\mathcal{M}}\right)^{*}=\mathcal{B}_{\mathcal{M}^{*}}^{*}
$$

where $\mathcal{B}^{*}$ is the unit ball of $\|\cdot\|^{*}$ and $\mathcal{B}_{\mathcal{M}^{*}}^{*}=$ $\bigcap_{S \in \mathcal{S}\left(\mathcal{M}^{*}\right)} S^{-1} \mathcal{B}^{*}$.

Remark 7.1: To reinterpret the statement of the previous Theorem 7.2 note that by Lemma 5.3 the ball $\mathcal{B}_{\mathcal{M}^{*}}^{*}$ is the unit ball of the Feller norm corresponding to $\mathcal{M}^{*}$ and the norm $\|\cdot\|^{*}$. Thus the result states that the dual of a convextransient norm for $\mathcal{M},\|\cdot\|$ is a Feller norm for $\mathcal{M}^{*},\|\cdot\|^{*}$.

Example 7.1: For the case $\mathbb{T}=\mathbb{R}_{+}$we consider the differential equation $\dot{x}=A x$ for the matrix $A=\left(\begin{array}{cc}-5 & 36 \\ 0 & -20\end{array}\right)$. Its initial growth rate with respect to the Euclidean norm is given by $\mu_{2}(A)=\frac{1}{2} \lambda_{\max }\left(A+A^{*}\right)=7$ hence the Euclidean norm is not a transient norm. Figure 2 shows the unit balls of the Feller norm, $\mathcal{B}$, and of the convex-transient norm, $\tilde{\mathcal{B}}$. Both unit balls are invariant under the flow of the system.

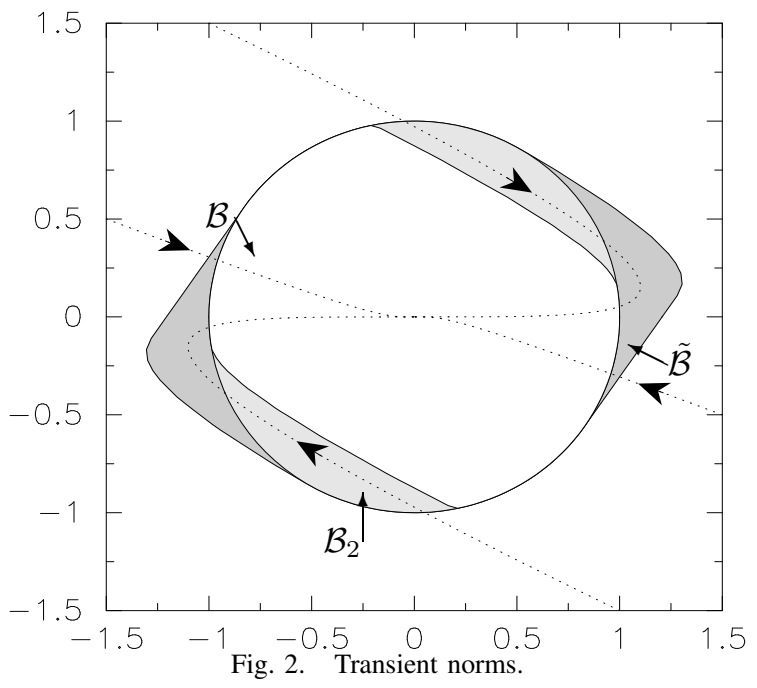

ACKNOWLEDGEMENT

This work was partially supported by the Enterprise Ireland grant 00/PI.1/C067. Elmar Plischke gratefully acknowledges support by the DFG grant HI 372/4.

\section{REFERENCES}

[1] G.-C. Rota and G. Strang, "A note on the joint spectral radius," Indag. Math., vol. 22, pp. 379-381, 1960.

[2] N. E. Barabanov, "Absolute characteristic exponent of a class of linear nonstationary systems of differential equations," Siberian Mathematical Journal, vol. 29, no. 4, pp. 521-530, 1988.

[3] F. Colonius and W. Kliemann, "Maximal and minimal Lyapunov exponents of bilinear control systems," J. Diff. Eqns., vol. 101, pp. 232-275, 1993

[4] N. E. Barabanov, "Lyapunov indicator of discrete inclusions. I-III," Autom. Remote Control, vol. 49, no. 2, pp. 152-157, no. 3, pp. 283287, no.5, pp. 558-565, 1988.

[5] V. S. Kozyakin, "Algebraic unsolvability of problem of absolute stability of desynchronized systems," Autom. Rem. Control, vol. 51, no. 6, pp. 754-759, 1990.

[6] F. Wirth, "The generalized spectral radius and extremal norms," Lin. Alg. Appl., vol. 342, pp. 17-40, 2002.

[7] _ - Stability theory of perturbed systems: Joint spectral radii and stability radii, ser. Lecture Notes in Mathematics. Berlin: SpringerVerlag, 2005, to appear.

[8] G. Dahlquist, "Stability and error bounds in the numerical integration of ordinary differential equations," Tekn. Högskol. Handl., vol. 130, 1959.

[9] S. M. Lozinskiǔ, "Error estimates for numerical integration of ordinary differential equations," Izv. Vyš̌. Učebn. Zaved Matematika, vol. 5, pp. 52-90, 1958, errata 5:222, 1959.

[10] M. I. Gil', Stability of Finite and Infinite Dimensional Systems. Boston/Dordrecht/London: Kluwer Academic Publishers, 1998.

[11] D. Hinrichsen and A. J. Pritchard, Mathematical Systems Theory I. Berlin: Springer-Verlag, 2005.

[12] V. Y. Protasov, "The joint spectral radius and invariant sets of linear operators," Fundam. Prikl. Mat., vol. 2, no. 1, pp. 205-231, 1996.

[13] W. Feller, "On the generation of unbounded semi-groups of bounded linear operators," Ann. of Math., Ser. 2, vol. 58, pp. 166-174, 1953.

[14] M. A. Berger and Y. Wang, "Bounded semigroups of matrices," Lin. Alg. Appl., vol. 166, pp. 21-27, 1992.

[15] T. Ström, "On logarithmic norms," SIAM J. Numer. Anal., vol. 12, no. 5, pp. 741-753, 1975 .

[16] M. Vidyasagar, Nonlinear Systems Analysis, 2nd ed. Englewood Cliffs, NJ: Prentice Hall, 1993.

[17] R. A. Horn and C. R. Johnson, Matrix Analysis. Cambridge University Press, 1990.

[18] R. T. Rockafellar, Convex Analysis. Princeton University Press, 1970.

[19] F. H. Clarke, Y. S. Ledyaev, R. J. Stern, and P. R. Wolenski, Nonsmooth Analysis and Control Theory, New York: Springer, 1998, vol. 178. 